\title{
Inspection of Wave Propagation Due to Partial Discharge Occurring in Current Transformer Using 3-D Finite Difference Time Domain Based Technique
}

\author{
R. M. Sharkawy and Tamer Adel Aziz
}

\begin{abstract}
This work aims to investigate the impact of a Partial Discharge (PD) pulse happening in the insulator conductor of a Current Transformer (CT) in a metallic tank filled with mineral oil. Finite Difference Time domain (FDTD, being an acceptable computation technique in the electromagnetic (EM) domain, is utilized for the simulation of the transient waves due to the PD pulse at different locations. The electric field intensity distribution at different planes is visualized. Analysis estimated both the optimal position of a sensor for PD detection and the dominant frequency enclosing the signals. Simulation run results are coded and recorded using Matlab. Observation of the PCOLOR outputs is discussed.
\end{abstract}

Index Terms-Current transformer (CT), electromagnetic (EM) waves, discharge (PD), finite difference time domain (FDTD), MATLAB

\section{INTRODUCTION}

Failure of a Current Transformer (CT) may cause incorrect current signals and lead to loss of supply. Therefore, it is important to detect early failures in CTs, so that they can be maintained or replaced. This in turn helps in saving the maintenance time and cost, and the overall reliability improvement of a power system.

Partial discharges (PD) are a major indication of the inception of insulation failure. They occur in the insulation systems and may have various natures and locations [1]. In addition to classical electrical measurement methods, PD is also accompanied by sound waves and electromagnetic waves at very high frequencies (300 $\mathrm{MHz}$ to $1500 \mathrm{MHz}$ ), this evoked nonconventional method like acoustic and ultra high frequency (UHF) to be employed for detection and localization. UHF technique has an advantage of increased PD detection and localization accuracy since the possibility of noise interference is almost eliminated. The challenges of PD detection circuits are the design of proper sensors (antennas) capable of signal detection in broadband range as well further signal interpretation. Thus, the platform is still inviting for researchers to devise novel techniques for the inspection of PD using UHF sensors as well as the choice of proper types and locations for those sensors for various apparatus.

Motivated by the success of the finite difference Time Domain (FDTD) technique for electromagnetic computation in many applications [2], it is utilized in this work to

Manuscript received February 12, 2012; revised March 16, 2012

The authors are with Arab Academy for Science, Technology and Maritime Transport, Electrical and Computer Control Engineering Depart, Cairo Egypt. simulate the electrical field which can be measured by sensors, when PD occurs inside transformers. The simulation of the electromagnetic wave propagation and its distribution intensity due a PD pulse incident at the centre of an insulated conductor is investigated.

\section{CURREnt Transfomer}

A Current Transformer forms an important part of the power network to monitor load currents for protection and measurement purposes. The equipment requires insulation to isolate the high voltage windings from the low voltage windings. The insulation is used to withstand both the steady state and transient state. It gradually deteriorates due to thermal, mechanical, electrical, and environmental stresses [3].

The structure of a CT has been chosen based on the design of a hairpin type current transformer [4, 5]. The standard design is a hermetically sealed expansion system with a nitrogen cushion compressed by thermal expansion of the oil. The cores and secondary windings are mounted in an aluminum tank. The insulator consists as standard of highgrade brown-glazed porcelain. The primary winding consists of one or more parallel conductors, which are insulated with a special paper which has high mechanical and dielectric strength, low dielectric losses and good resistance to ageing. The main part used for simulation focus on the high voltage terminal.

The space for simulation can be restricted by transformer tank as conducting boundary condition in isotropic media. To investigate the field inside and outside a transformer, a small part of the transformer tank placed in air is modeled like the situation in the real world. Meanwhile, the escaped energy of the waves and transients at different planes are observed. The most suitable places for positioning the sensor are discussed later in the paper.

FDTD technique is used in the EM computation of the field propagated in time and space at the desired locations of the transformer and discussed below.

\section{FINITE DIFFERENCE TIME DOMAIN TECHNIQUE}

The FDTD method utilizes the central difference approximation to discretize the two Maxwell's curl equations, namely, Faraday's and Ampere's laws, in both the time and spatial domains, and then solves the resulting equations numerically to derive the electric and magnetic field distributions at each time step using an explicit leapfrog scheme. The FDTD solution, thus derived, is second order accurate, and is stable if the time step satisfies 
the Courant condition.

In Yee's scheme [6], the computational domain is discretized by using a rectangular grid. The FDTD algorithm [7] constructs a solution to the following two Maxwell's curl equations:

$$
\begin{aligned}
\nabla \times \vec{E} & =-\mu \frac{\partial \vec{H}}{\partial t} \\
\nabla \times \vec{H} & =-\delta \vec{E}+\varepsilon \frac{\partial \vec{E}}{\partial t}
\end{aligned}
$$

Mur first-order absorbing boundary condition is used to truncate the tangential components of $\mathrm{E}$ at the boundary. In the other hand, the interfaces are handled carefully between different media in an inhomogeneous environment. A separate weighted average is taken in consideration respectively during the $\mathrm{E}-\mathrm{H}$ fields calculations at these interfacing points [8].

As per [9], the initial rise-time of the current waveform produced by a PD is sufficiently high to cause the frequency spectrum to extend into the radio frequency region $(30 \mathrm{KHz}$ - $300 \mathrm{GHz}$ ), which corresponds to the frequency of electrical signals normally used to produce and detect radio waves. As a consequence, a proportion of the PD energy is radiated into the free-space adjoining the site of the discharge. The electromagnetic radiation is related to the rate of change of current and the structure and connections of the radiated. For this reason PD can be detected using wideband radiometric antennas [10], [11]. The challenges of PD detection circuits are the design of proper sensors (antennas) capable of signal detection in broadband range and the signal interpretation circuits [12], [13].

$$
E_{z} \leftarrow E_{z}+\frac{\Delta t}{\varepsilon} \cdot \frac{I_{z}(t)}{\Delta x \Delta y}
$$

In this simulation a Gaussian current pulse is set at the Zdirectional as PD source given by [14], [15]:

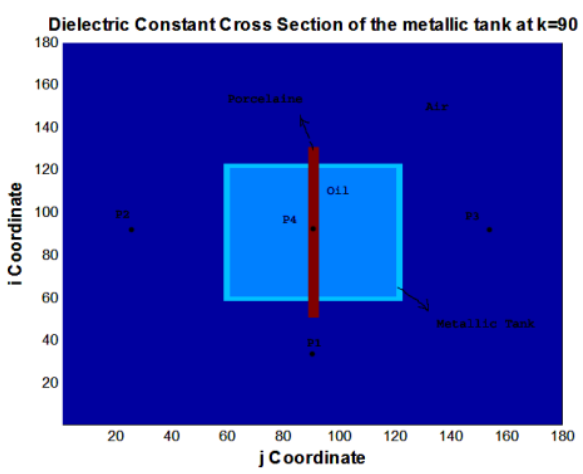

Fig. 1. Simplified current transformer structure.

$$
i(t)=\frac{I}{T_{r}} t e^{\left(1-t / T_{r}\right)}=\frac{q}{e^{2} T_{r}^{2}} t^{\left(1-t / T_{r}\right)}
$$

In FDTD code each iteration includes additional operation for the computation of the new E field. PD current can be converted to the initial electric field in $\mathrm{z}$ direction by using (3). This approach has been validated for a cylindrical coordinate system and representing a soft type of source [16].

\section{Simulation Space}

The analysis of the electromagnetic field distribution relevant to a PD pulse within a current transformer has been conducted through an FDTD simulation. A simplified model, driven from the CT structure described above, is outlined in Fig 1. This model is composed of the inner conductor and a metal cap.

The simulated volume was $0.9 \times 0.9 \times 0.9 . \mathrm{m}$ box. A $5 \mathrm{~mm}$ rectangular mesh was applied, giving a total of $180 \mathrm{x} 180 \mathrm{x}$ 180 cells with absorbing boundaries condition. The time step was automatically set to be 9.6292 ps defined by the CLF stability criterion.

$$
\Delta \mathrm{t} \leq \frac{\Delta}{\mathrm{c} \sqrt{3}}
$$

The impulsive signals obtained are based around a $15.8 \mathrm{~ns}$ wave simulation, which roughly consumes 3 hours and 35 minutes on a computer with $2.21 \mathrm{GHz}$ processor Core 2 Duo with 3 GB RAM. Larger space and smaller mesh can be set up, resulting in more calculations and longer simulation time. However, the settings will be eventually subject to the capability of the computer and the number of bit handling capabilities of Matlab.

The simulation of CT housing is conducted. The dielectric medium inside the volume is air. The CT cap is modeled using a conducting tank with dimension $0.32 \times$ $0.32 \times 0.32$ meters, which is in the centre of the simulation volume. The CT simulated is filled with mineral oil. Two holes of $0.02 \times 0.02$ meters are located along the $\mathrm{X}$-axis on both sides of the CT tank through which a 0.4 m length bar simulating the insulator conductor in the CT housing.

TABLE I: Simulation MATERIAL ElectricAl PROPERTIES

\begin{tabular}{|l|l|l|l|}
\hline \multirow{2}{*}{$\#$} & \multicolumn{3}{|c|}{ Material Electrical Properties } \\
\cline { 2 - 4 } & \multicolumn{1}{|c|}{ Material } & \multicolumn{1}{c|}{$\boldsymbol{\epsilon}_{\boldsymbol{r}}$} & \multicolumn{1}{c|}{$\boldsymbol{m}^{\mathbf{- 1}}$} \\
\hline 1 & Air & 1 & $0.5 e^{-2}$ \\
\hline 2 & Aluminum & 2.2 & $0.377 e^{-2+8}$ \\
\hline 3 & Mineral Oil & 2.1 & $8 e^{-2}$ \\
\hline 4 & Insulation & 5.5 & 0 \\
\hline
\end{tabular}

Table I lists the electrical properties of above simulated domain. Where $\epsilon_{\mathrm{r}}$ and $\delta$ are the relative permittivity and electrical conductivity respectively of a material. In the simulation, a current source oriented in the $\mathrm{z}$-direction is used as a PD source, and is located at the centre of the tank and the conductor. The coordinate of the current source are $(0.45 \mathrm{~m}, 0.45 \mathrm{~m}$, and $0.45 \mathrm{~m})$.

\section{Simulation Result AND Discussion}

Four different planes were investigated. Plane 1 is located on the centre of the $\mathrm{y}-\mathrm{z}$ plane at $\mathrm{x}=0.75 \mathrm{~m}$, which is the nearest position to the end of the insulated conductor. Plane 2 are located at the centre of the $\mathrm{x}-\mathrm{z}$ plane at $\mathrm{y}=0.75 \mathrm{~m}$. Plane 4 is located at $\mathrm{x}-\mathrm{z}$ plane at $\mathrm{y}=0.45 \mathrm{~m}$. This equates to $0.3 \mathrm{~m}$ from the source point at either side in vertical plane except for Plane 4 which was at the centre of simulated volume. Fig $2-8$ show the $E_{z}$ field intensity distribution at these planes at different time instants. 
It is well recognized by observing the distribution intensity of the $E_{z}$ radiation that the insulation, in the presence of a PD signal, deteriorates and allows the propagation of the wave. Its main function becomes corrupted and leads the CT to break down.

The Field components $E_{z}, E_{y}$ and $E_{x}$ were investigated at four different Positions. Position 1 is located in the centre of the $\mathrm{y}-\mathrm{z}$ plane at $\mathrm{x}=0.75 \mathrm{~m}$, which is the nearest position to the end of the insulated conductor. Position 2 and 3 are located at the centre of the $\mathrm{x}-\mathrm{z}$ plane at $\mathrm{y}=0.15 \mathrm{~m}$ and $\mathrm{y}=$ $0.75 \mathrm{~m}$. Position 4 is located at $\mathrm{x}-\mathrm{y}$ plane at $\mathrm{z}=0.75 \mathrm{~m}$. This equates to $0.3 \mathrm{~m}$ from the source point at either side in vertical plane. Fig. 1 illustrates those positions, whose coordinates are listed in Table II.

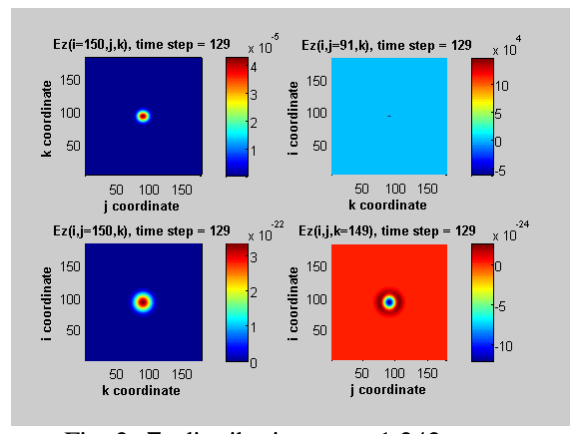

Fig. 2. $\boldsymbol{E}_{\boldsymbol{z}}$ distribution at $\mathrm{t}=1.242 \mathrm{~ns}$

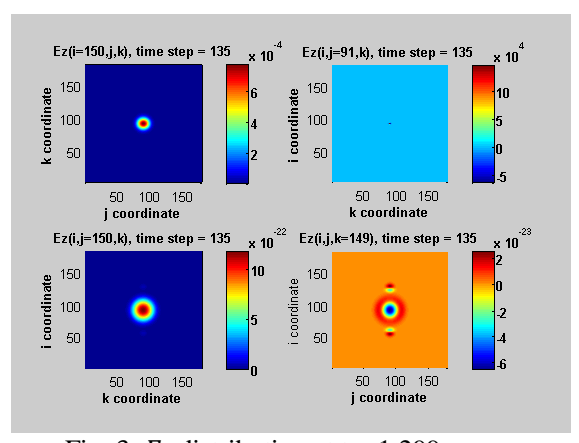

Fig. 3. $E_{z}$ distribution at $\mathrm{t}=1.299 \mathrm{~ns}$

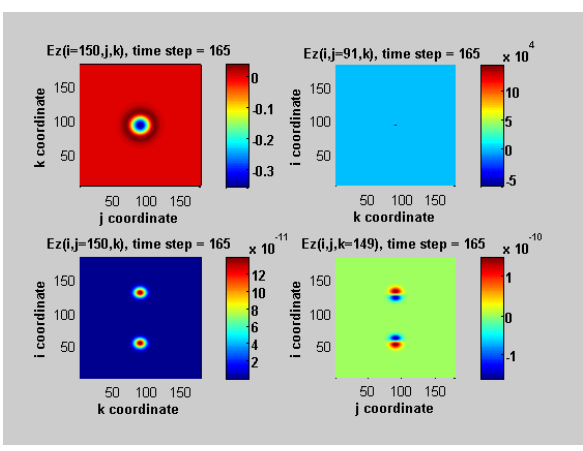

Fig. 4. $\mathrm{E}_{\mathrm{z}}$ distribution at $\mathrm{t}=1.588 \mathrm{~ns}$

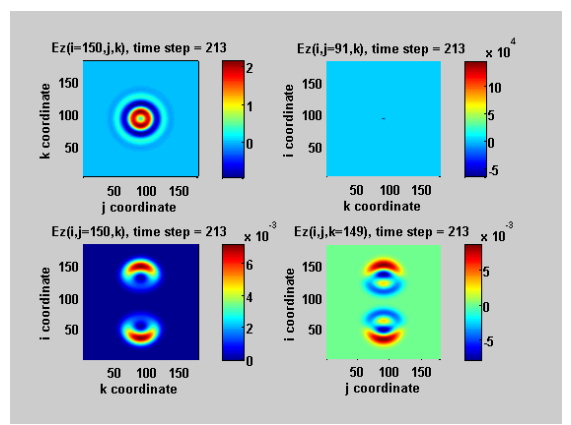

Fig. 5 . $\mathrm{E}_{\mathrm{z}}$ distribution at $\mathrm{t}=2.051 \mathrm{~ns}$

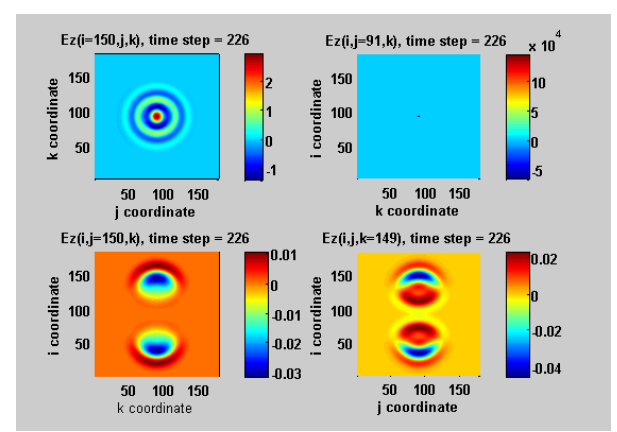

Fig. $6 . \mathbf{E}_{\mathbf{z}}$ distribution at $\mathrm{t}=2.176 \mathrm{~ns}$

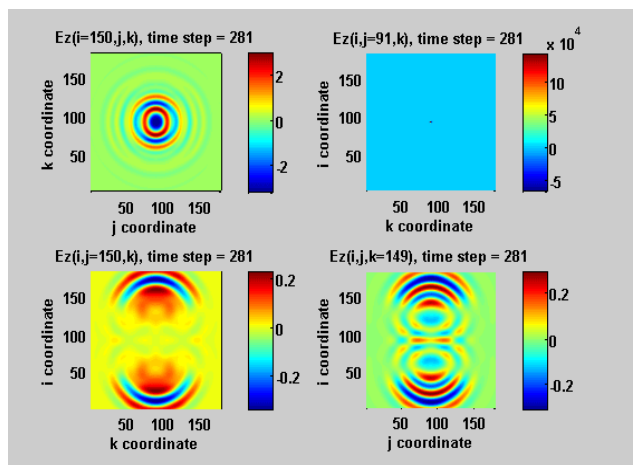

Fig. 7. $\mathbf{E}_{\mathrm{z}}$ distribution at $\mathrm{t}=2.705 \mathrm{~ns}$



Fig. 8. $\mathbf{E}_{\mathbf{z}}$ distribution at $\mathrm{t}=7.202 \mathrm{~ns}$

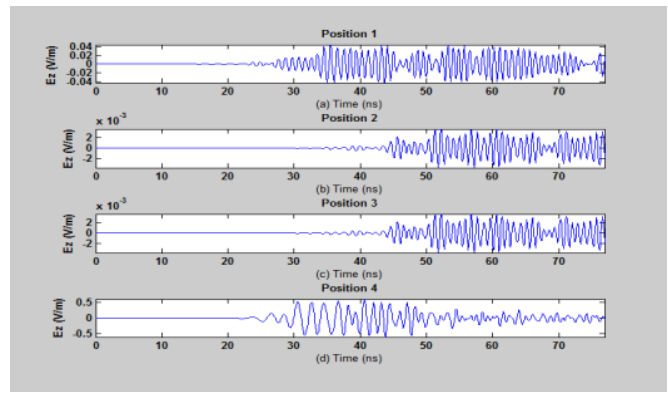

Fig. 9. $\mathbf{E}_{\mathbf{z}}$ component detection at the four positions.

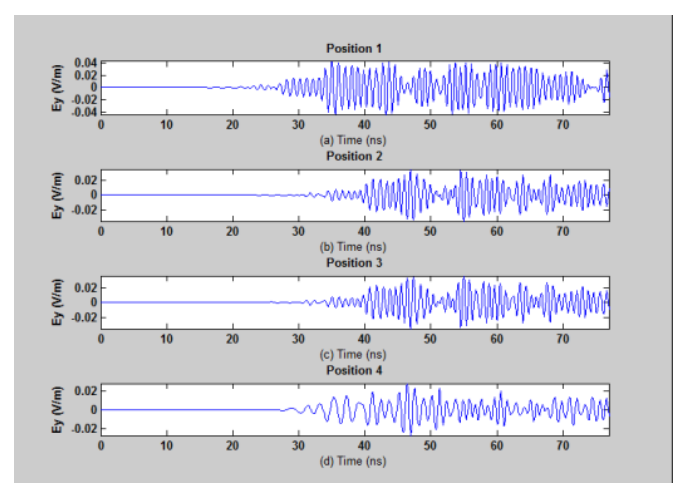

Fig. 10. $\mathbf{E}_{\mathbf{y}}$ component detection at the four positions. 
TABLE II: THE COORDINATES OF THE TREE POSITIONS

\begin{tabular}{|l|l|}
\hline \multicolumn{1}{|c|}{ Position \# } & \multicolumn{1}{c|}{ Coordinates $(\mathbf{x}, \mathbf{y}$ and $\mathbf{z})$} \\
\hline 1 & $(0.75 \mathrm{~m}, 0.45 \mathrm{~m}, 0.45 \mathrm{~m})$ \\
\hline 2 & $(0.15 \mathrm{~m}, 0.45 \mathrm{~m}, 0.45 \mathrm{~m})$ \\
\hline 3 & $(0.45 \mathrm{~m}, 0.75 \mathrm{~m}, 0.45 \mathrm{~m})$ \\
\hline 4 & $(0.45 \mathrm{~m}, 0.45 \mathrm{~m}, 0.75 \mathrm{~m})$ \\
\hline
\end{tabular}

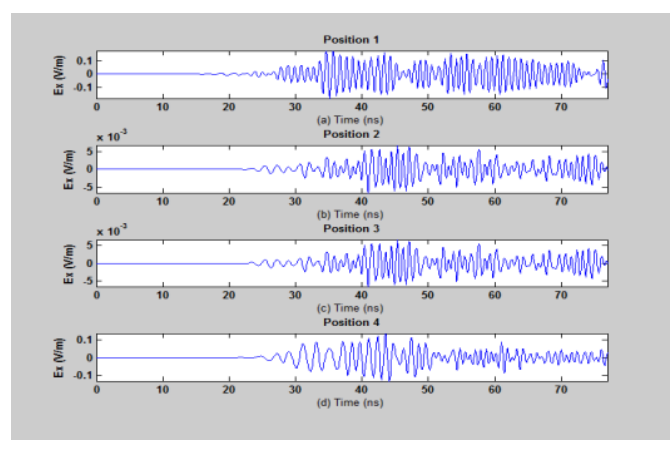

Fig. 11. $\mathbf{E}_{\mathbf{x}}$ component detection at the four positions.

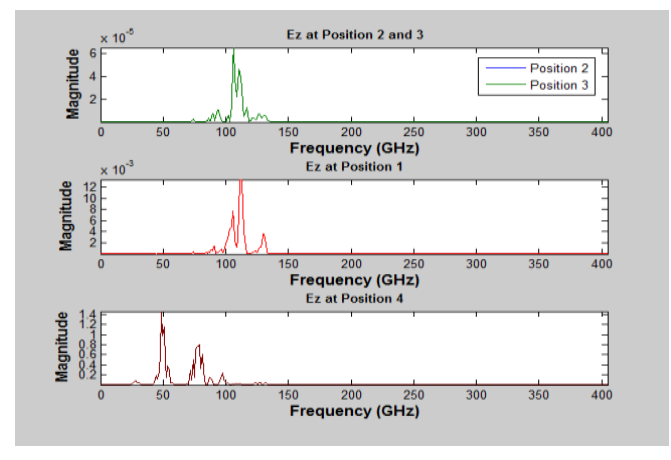

Fig. 12. $\mathbf{E}_{\mathbf{z}}$ frequency spectrum at the four positions.

TABLE III: THE MAXIMUM AMPLITUdE OF THE E FIELDS AT THE FOUR POINTS

\begin{tabular}{|c|l|c|c|c|}
\hline \multirow{2}{*}{$\begin{array}{c}\text { Position } \\
\#\end{array}$} & \multicolumn{4}{|c|}{ The MAX amplitude of the E fields } \\
\cline { 2 - 5 } & $\mathbf{1}$ & $\mathbf{2}$ & $\mathbf{3}$ & $\mathbf{4}$ \\
\hline $\boldsymbol{E z}(\boldsymbol{V} / \boldsymbol{m})$ & 0.0443 & 0.0037 & 0.0037 & 0.6106 \\
\hline $\boldsymbol{E y}(\boldsymbol{V} / \boldsymbol{m})$ & 0.0427 & 0.0355 & 0.0357 & 0.028 \\
\hline $\boldsymbol{E x}(\boldsymbol{V} / \boldsymbol{m})$ & 0.179 & 0.0067 & 0.0067 & 0.137 \\
\hline
\end{tabular}

The electric filed components are monitored to see how each signal shape goes with time, analyze the magnitudes and dominant frequency to understand where to mount an external antenna making it most responsive to the polarised field.

Fig. 9 to 11 shows the time wave form of the fields components $\mathrm{E}_{\mathrm{z}}, \mathrm{E}_{\mathrm{y}}$ and $\mathrm{E}_{\mathrm{x}}$ respectively. At position 1, the nearest to the insulator conductor, all the fields were leading the other two positions in the term of detection. The amplitude of the $\mathrm{E}$ components at position 1 was the highest compared to the amplitude of the rest positions. The E fields detected at position 2 and 3 of each component were exactly similar in shape and amplitude. It is also quite notable that at position 1, the E fields components are all having the same shape. $E_{z}$ and $E_{y}$ were having the same amplitude compared to $E_{x}$ which was having the highest amplitude. At position $4, \mathrm{E}_{\mathrm{z}}$ amplitude was the highest because the PD input signal was z-directed. Table III lists the maximum amplitude of electric fields at three positions.

The frequency spectrum of the $E_{z}$ component was investigated. Fig 12 shows the dominate frequency detected per position. The frequency of position 2 and 3 are superposed as these two positions are symmetrically located. Both enclose a dominant frequency of $106 \mathrm{GHz}$ while at position 1 the dominant frequency was reading $112 \mathrm{GHz}$. At position 4 , the dominant frequency was down to the half. It reads $50 \mathrm{GHz}$. The amplitude of the frequency was relatively detected high at position 1 which is nearest to the insulator compared to position 2 and 3 . The amplitude of position 3 was the highest.

\section{CONCLUSION}

An investigation into electromagnetic radiation of partial discharge has been performed incorporating simulation with FDTD Technique. The propagation waves are radiated outside of the CT mainly through the bar. It can be highly suggested that the sensor receiving the PD signal outside a CT should be mounted near the primary conductor for obtaining maximum signal amplitude which has in parallel the highest frequency component among the others.

Proposed future work for this study can include developing a more detailed model for the current transformer as well as the transfer function of the detectors antennas in the simulation algorithm. Moreover, the use of the Time Difference of Arrival (TDOA) approach for PD localization can be investigated.

\section{REFERENCES}

[1] F. H. Kreuger, "Discharge Detection in High Voltage Equipment," Butterworths, London, 1989.

[2] D. Pommerenke, R. Jobava, and R. Heinrich, "Numerical Simulation of Partial Discharge Propagatiion in Cable Joints using the Finite Difference Time Domain Method," IEEE Electrical Insulation Magazine, p.6-11, Vol. 18, no. 6, December 2002.

[3] H. Borsi, 'The relation between thermal and electrical stress and the PD behavior of epoxy resin in transformers," IEEE Trans. Electr. Insul. vol. 28, No. 6, pp. 1007-1015, Dec. 1993.

[4] "ABB Outdoor Instrument Transformers Design Features and Advantages," ABB Inc, Mar. 2006.

[5] S. Xiao, P. J. Moore, and M. D. Judd, Strathclyde Univ., Glasgow; "Time Frequency Modeling of Partial Discharge Radiation from Current Transformers," in Proc. of the 41st International Universities Power Engineering Conference, 2006. UPEC '06.

[6] K S Yee, "Numerical solution of initial boundary value problems involving Maxwell's equations in isotropic media," IEEE Trans. Antennas and Propagation, Vol. AP-14, No. 3 pp. 302-307, May 1966.

[7] A Taflove, "Computational electrodynamics: The finite difference time-domain method," Artech House, 1995.

[8] W. Yu, R. Mittra, T. Su, Y. Liu, and X. Yang; "Parallel FiniteDifference Time-Domain Method," Artech House, 1995.

[9] S. Xiao, P. J Moore, M. D. Judd, Portugues, I.E. "An Investigation into Electromagnetic Radiation due to Partial Discharges in High Voltage Equipment. Power Engineering Society General Meeting, IEEE 2007.

[10] S. D. Meier, P. J. Moore, and P. F. Coventry, "Finite element modeling of circuit breaker switching emissions' propagation in GIS," in Proc. of 2005 International Symposium on Electrical Insulating Materials, Japan, June, 2005.

[11] P. Moore, I. Portugues, and I. Glover, "Radiometric Location of Partial Discharge Sources on Energized High-Voltage Plant," IEEE Transactions on Power Delivery, Vol.20, No.3, July 2005.

[12] C. Abraham and S.V. Kulkarni, "FDTD Simulated Propagation of Electromagnetic Pulses due to PD for Transformer Diagnostics," TENCON 2008 - 2008 IEEE Region 10 Conference.

[13] T. Hoshino, S. Maruyama, and T. Sakakibara, "Simulation of Propagating Electromagnetic Wave due to Partial Discharge in GIS 
using FDTD," IEEE TRANSACTIONS ON POWER DELIVERY, VOL. 24, NO. 1, JANUARY 2009.

[14] Y. Tian, M. Kawada, and K. Isaka, "Visualization of Electromagnetic Waves Emitted from Multiple PD Sources on Distribution Line by Using FDTD Method," in Proc. of 2008 International Symposium on Electrical Insulating Materials, September 7-11, 2008, Yokkaichi, Mie, Japan

[15] B. Xu, J. Li, W. Si, and Y. Li, "Simulating Propagation Characteristic of UHF Signal for PD Monitoring in Transformers by FDTD Technique," Power and Energy Engineering Conference, 2009. APPEEC 2009. Asia-Pacifi

[16] K. Yoshitomi; "A Study on Current Sources used in Finite-Difference Time-Domain Antenna Analysis," Applied Electromagnetics Conference, 2007. AEMC 2007. IEEE

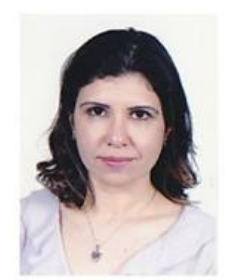

R. M. Sharkawy received her BSc and MSC from Ein Shams, Egypt, all in electrical engineering in 1993, 1997, and 2002 respectively. She worked as a research assistant in the faculty of engineering at the United Arab Emirates University (1993-1996). She was a research scientist at the voltage laboratory of the national institute for standards, Giza, Egyp (1996 - 2006). Currently she is the assistant deans for technical affairs and a faculty member at the electrical $\mathrm{n}$ control engineering department at the Arab Academy for
Science and Technology and Maritime Transport, Cairo, Egypt. All research interest in the area of metrology, high voltage measurements and the applications of intelligent techniques for the assessment of the insulation systems.

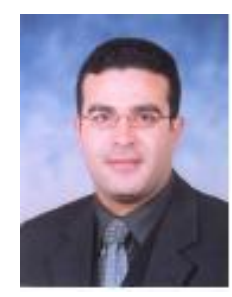

Tamer Adel Aziz is born in Cairo, Egypt in 1977. $\mathrm{He}$ was awarded the degree of Bachelor of Science in electrical and computer engineering from the Higher Technological Institute of the $10^{\text {th }}$ of Ramadan City, Egypt in January 2001. He held several positions within Orange Business Services, France Telecom Group Inc, in Cairo, Egypt. He occupied the position of the INSTALLATION SUPPORT ENGINERR starting from $15^{\text {th }}$ of Feb 2004 till end of 2006. He also held the position of the TELECOM SOURCING BUYER till the $1 \mathrm{R} 0^{\text {th }}$ of Dec 2010 . He is currently a PROJECT MANAGER in the VOICE SEVICES AND FRAUD area Mr. Tamer is Cisco Internetworking Professional Certified (CCIP), and Information Technology Infrastructure Library (ITIL foundation) Certified. He is currently working in his Master Degree 